\section{Enfermedad de Hirschsprung y apendicitis. Una asociación rara}

\author{
Baeza-Herrera $\mathrm{C}^{1}$, López-Medina $\mathrm{P}^{2}$, Vidal-Medina $\mathrm{JM}^{2}$, Cruz-Viruel $\mathrm{N}^{3}$, \\ Velasco-Soria $\mathrm{L}^{4}$
}

\section{Resumen}

La enfermedad de Hirschsprung asociada con apendicitis es una condición poco usual; suman 15 los reportados en la literatura, ya incluido este caso. Se informa el caso de un lactante de 12 meses de edad que ingresó con fiebre, anorexia, distensión abdominal, vómito, evacuaciones hemáticas y antecedente de estreñimiento. Se realizó laparotomía exploradora que reportó apendicitis y megacolon congénito o enfermedad de Hirschsprung. El reporte histopatológico reveló periapendicitis, huevecillos de áscaris lumbricoides, con ausencia de células ganglionares.

PALABRAS CLAVE: enfermedad de Hirschsprung, apendicitis, constipación.

Acta Pediatr Mex. 2016 May;37(3):159-164.

\section{Hirschsprung's disease and appendicitis. A rare association.}

Baeza-Herrera $\mathrm{C}^{1}$, López-Medina $\mathrm{P}^{2}$, Vidal-Medina $\mathrm{JM}^{2}$, Cruz-Viruel $\mathrm{N}^{3}$, Velasco-Soria $\mathrm{L}^{4}$

\begin{abstract}
Hirschsprung's disease associated with appendicitis is a rare condition, since this case included total 15 those reported in the literature. For infants 12 months of age income with fever, anorexia, abdominal distension, vomiting, blood evacuations and history of constipation is reported. Exploratory laparotomy was performed finding appendicitis and congenital megacolon or Hirschsprung disease. The histopathological report revealed periappendicitis, roundworm eggs with absence of ganglion cells.
\end{abstract}

KEYWORDS: Hirschsprung disease; appendicitis; constipation
${ }^{1}$ Jefe de la División de Cirugía. Hospital Pediátrico Moctezuma. Secretaría de Salud del Distrito Federal. Profesor Titular de Cirugía Pediátrica y del Curso para Postgraduados de Alta Especialidad en Cirugía del Recién Nacido. Universidad Nacional Autónoma de México.

${ }^{2}$ Residente de Cirugía.

${ }^{3}$ Patóloga adscrita, Unidad de Patología Hospital Juárez de México.

${ }^{4}$ Cirujano adscrito y profesor ayudante.

Recibido: 14 de junio 14

Aceptado: 15 de marzo del 2016

Correspondencia

Dr. Carlos Baeza Herrera.

Oriente 158 No. 189

Colonia Moctezuma, 2a Sección

15530 Ciudad de México

Tel: 57622421 / 55714057

dr.carlosbaeza@yahoo.com.mx

Este artículo debe citarse como

Baeza-Herrera C, López-Medina P, Vidal-Medina JM, Cruz-Viruel N, Velasco-Soria L. Enfermedad de Hirschsprung y apendicitis. Una asociación rara. Acta Pediatr Mex 2016;37(3):159-164. 


\section{INTRODUCCIÓN}

Muy temprano en la gestación, el sistema nervioso primitivo (de manera normal y estando en la etapa de cresta neural) y las neuronas ahí generadas extienden la inervación hacia la totalidad del tubo digestivo, culminando esta fase del desarrollo entre las $10^{a}$ y $12^{a}$ semanas de gestación. Una vez distribuida la red nerviosa, que a la postre formará los plexos submucoso y mientérico, éstos se encargarán de la inervación intrínseca del tubo digestivo que a su vez, entre otras funciones, propiciará que la motilidad intestinal sea efectiva. Sin embargo, cuando por alguna razón la distribución de las ramificaciones de los plexos se interrumpe, aparecen alteraciones en los movimientos propulsivos del colon, lo que clínicamente se hace manifiesto mediante la constipación, síntoma cardinal de la enfermedad. La agangliosis congénita o enfermedad de Hirschsprung se caracteriza por la detención en fases iníciales de la embriogénesis de la migración neuronal céfalo-caudal donde el tubo digestivo despliega el sistema nervioso periférico responsable de la inervación normal. ${ }^{1,2}$ Pueden existir otras enfermedades que se asocian por el origen embriológico como neuroblastoma y el síndrome de Waardenburg, que se caracteriza por alteraciones en el metabolismo de la melanina y se traduce en hipopigmentación cutánea y del pelo, así como distopia cantorum y sordera congénita. ${ }^{3}$

En la enfermedad de Hirschsprung existen casos donde se pueden asociar anomalías estructurales como atresia intestinal, ano imperforado y malrotación intestinal, perforación del colon y problemas infecciosos como la apendicitis aguda. ${ }^{4}$

\section{PRESENTACIÓN DE CASO CLÍNICO}

Lactante masculino de 12 meses de edad que inició su padecimiento dos días antes de su ingreso por presentar vómito en 10 ocasiones, distensión y dolor abdominal intermitente, presencia de sangre en evacuaciones, irritabilidad y fiebre de $38^{\circ} \mathrm{C}$. Como antecedente de importancia desde el nacimiento padeció estreñimiento que, en múltiples ocasiones, requirió del uso de supositorios. La primera evacuación en el periodo neonatal sucedió hasta el tercer día de vida.

A la exploración física se encontró en malas condiciones generales: pálido, hipoactivo, hiporreactivo y deshidratado, abdomen distendido, dolor generalizado, rebote positivo, peristalsis disminuida, cardiopulmonar sin compromiso, al tacto rectal se observó salida de abundante gas y materia fecal líquida y escasa, sin sangre, resto de la exploración normal. Los estudios de laboratorio reportaron leucocitos de 3,900, neutrófilos $25 \%$, linfocitos $49.9 \%$, hemoglobina de $9.9 \mathrm{~g} /$ $\mathrm{dL}$, hematocrito de $29.4 \%$, plaquetas 46,0000 , tiempo de protrombina de 17 segundos, tiempo de tromboplastina parcial de 69 segundos, sodio $138 \mathrm{mEq} / \mathrm{L}$, potasio $4.8 \mathrm{mEq} / \mathrm{L}$ y cloro $103 \mathrm{mEq} / \mathrm{L}$. La radiografía simple de abdomen reveló múltiples niveles hidroaéreos, opacidad pélvica sin aire libre subdiafragmático (Figura 1). El colon por enema mostró disminución del calibre del recto y presencia del "borde en serrucho" y la radiografía de control de 48 horas con medio de contraste e imagen de "borde de serrucho" aun visibles (Figura 2).

Dadas las condiciones del menor y con diagnóstico de apendicitis aguda versus enterocolitis por enfermedad de Hirschsprung, se realizó laparotomía exploradora mediante incisión de McBurney encontrando apéndice cecal gangrenado, membranas fibrinopurulentas, dilatación importante del intestino proximal, por lo que se tomaron biopsias a 5, 10, 15 y $20 \mathrm{~cm}$ de la deflexión peritoneal sin complicaciones.

La evolución posoperatoria fue desfavorable ya que persistía con datos de obstrucción intestinal, 


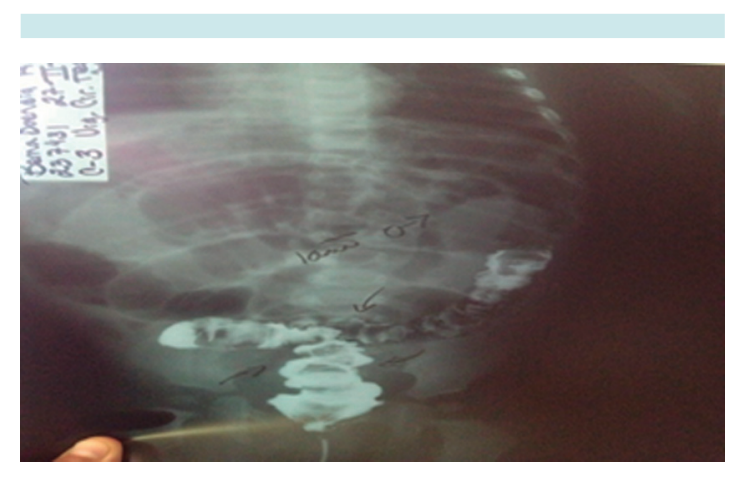

Figura 1. Radiografía con medio de contraste a las 48 horas, que muestra opacidad distal, visualización y persistencia del material yodado, así como niveles hidroaéreos generalizados, destacando el de la fosa ilíaca derecha.

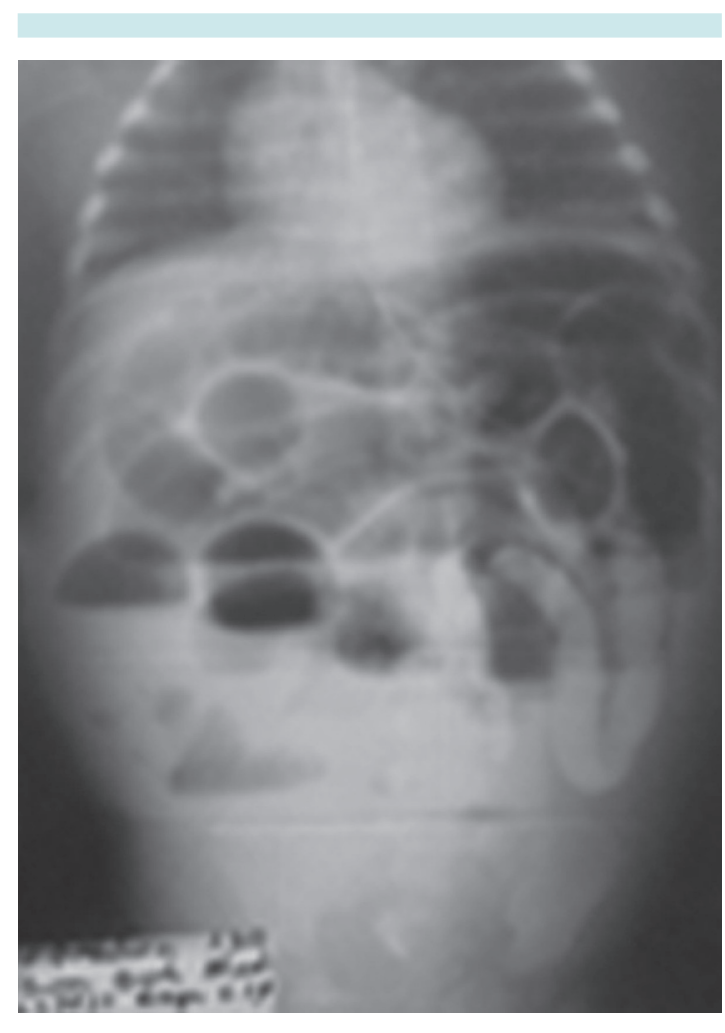

Figura 2. Colon por enema en su fase de eliminación que revela el "borde en serrucho" descrito en algunos textos como muy sugestivo de enterocolitis por enfermedad de Hirschsprung. por lo que se decidió la reintervención encontrando adherencias generalizadas y adenitis mesentérica, así como zona de estrechez en íleon terminal a $10 \mathrm{~cm}$ de la válvula ileocecal. Se efectuó lisis de adherencias, descompresión manual y obtención de biopsia ganglionar mesentérica.

La evolución del menor fue mala, persistiendo con patrón pulmonar restrictivo debido a la distensión abdominal, caracterizado por taquipnea, hipertensión arterial y edema generalizado. Dada la evolución fue nuevamente sometido a laparotomía con diagnóstico de enfermedad de Hirschsprung, encontrándose dilatación importante del íleon terminal, válvula ileocecal fibroedematosa por lo que se practicó ileostomía; evolucionando con remisión de la respuesta inflamatoria sistémica aguda por lo que se le dio la vía oral al séptimo día de postoperatorio.

El estudio histopatológico reportó ausencia de células ganglionares en recto sigmoides. El resultado de los estudios de imnunohistoquímica (P-100 y cromogranina) fueron compatibles con enfermedad de Hirschsprung (Figuras 3 y 4). Histológicamente el ganglio mostraba hipertrofia folicular y el apéndice, peri-apendicitis y huevecillos de áscaris lumbricoides.

El menor fue dado de alta a las dos semanas de haber ingresado. Actualmente está en preparación para efectuársele la operación definitiva de la agangliosis y se le dio tratamiento específico contra la ascariasis.

\section{ANÁLISIS}

La inervación del tubo digestivo se inicia antes de la $12^{\text {a }}$ semana de gestación con la aparición sobre la cresta neural de células nerviosas, las que mediante migración a través de los troncos neurales, arriban al espesor de la pared del tubo digestivo. Una vez instituida la red nerviosa, ésta 


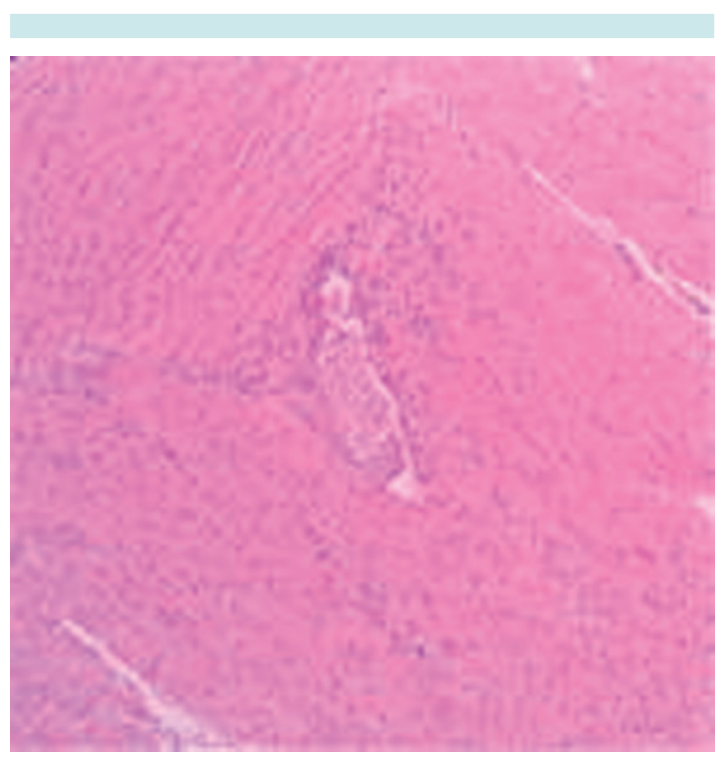

Figura 3. Estudio inmuno-histoquímico con la S100, negativo a células ganglionares.

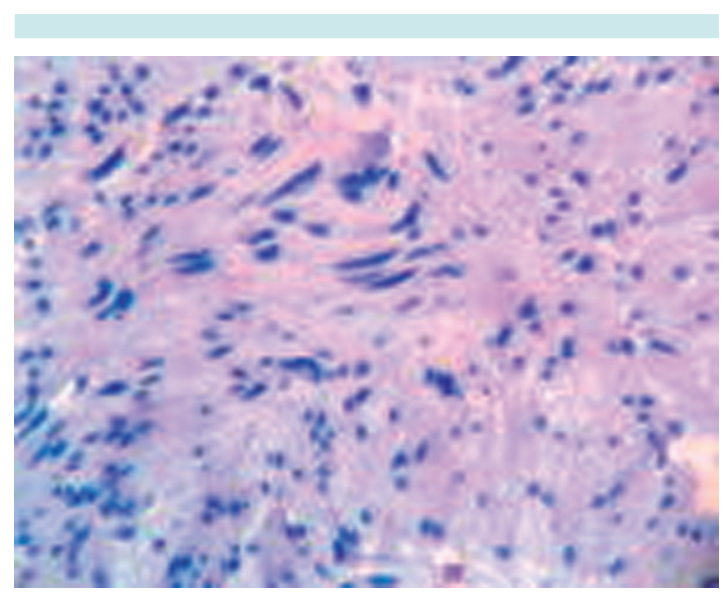

Figura 4. Estudio inmuno-histoquímico con cromogranina, igualmente negativo a células ganglionares.

se disemina iniciando en esófago en dirección cefalo-caudal, Ilegando entre la $10^{\mathrm{a}}$ y $12^{\mathrm{a}}$ semanas de gestación a las porciones terminales del colon. La presencia de los plexos submucoso y mientérico da al tubo digestivo la capacidad de efectuar movimientos propulsivos y de vacia- miento, mediante la instalación de fenómenos que conforme el individuo se desarrolla, van adquiriendo la madurez necesaria.

La agangliosis congénita o enfermedad de Hirschsprung se define como una alteración funcional del tubo digestivo y se caracteriza por movimientos peristálticos inefectivos debido a la ausencia de los plexos de Meissner y Auerbach en el colon distal. Las manifestaciones clínicas que presentan estos pacientes son obstrucción intestinal al nacimiento, vómito, distensión abdominal, ausencia de evacuaciones o la falta de evacuación en las primeas 24 horas de vida extrauterina. Puede presentarse con síntomas moderados como anorexia, dolor intermitente por semanas o meses, red venosa colateral y constipación seguida de obstrucción intestinal aguda. ${ }^{5}$

La sospecha diagnóstica se establece mediante el cuadro clínico y con base en los estudios radiológicos que muestran dilatación intestinal, múltiples niveles hidro-aéreos, opacidad pélvica total y el colon por enema suele revelar una área de estrechamiento denominada "zona de transición", la que puede persistir visible hasta 48 horas después de la impregnación. Sin embargo, el diagnóstico de enfermedad de Hirschsprung se establece mediante estudio histopatológico, caracterizado por la ausencia de células ganglionares.

La apendicitis aguda en los lactantes es una entidad poco frecuente, el cuadro clínico puede presentarse en forma atípica, por esta razón existe una alta incidencia de apendicitis complicadas. Puede presentarse con fiebre, ataque al estado general, vómito, diarrea y distensión abdominal, diagnosticándose generalmente por el médico de primer contacto como gastroenteritis o infección de vías urinarias la cual tratan con antibióticos, los cuales modifican aún más el cuadro clínico y retrasan el tratamiento quirúrgico. 
Cuando la apendicitis se presenta en lactantes con agangliosis congénita, no existe el antecedente de diarrea, vómito, ni distensión abdominal y la afectación es más bien de la serosa y no del espesor total de la pared apendicular. ${ }^{6,7}$

Se ha observado que cuando se asocia a enfermedad de Hirschsprung a apendicitis no existe destrucción ni inflamación de la pared apendicular, sólo se encuentra inflamación de la serosa a lo que denominan periapendicitis.

En los pacientes que presentan apendicitis con enfermedad de Hirschsprung suele haber neumo-peritoneo, por lo que cuando existe este hallazgo debe sospecharse la existencia de la asociación. ${ }^{7}$ De acuerdo con lo referido por Arlis y su grupo ${ }^{8}$ la apendicitis que acompaña a la agangliosis congénita debe considerarse como una entidad distinta ${ }^{9}$ (Cuadro 1).

Wagensteen y sus colaboradores ${ }^{11}$ mencionan que esta asociación podría explicarse porque al no poder dilatarse el segmento aganglionar, el contenido intra-colónico proximal aumenta en tanto que en segmentos distales al apéndice cecal se incrementa la presión intraluminal. De esa manera, si la válvula ileocecal es competente, la presión intra-colónica puede aumentar más rápidamente con volúmenes menores y así predisponer la perforación del apéndice. Otro factor que influye es que la válvula de Gerlach del apéndice cecal posee musculatura, por lo que al hacerla más competente, atrapa cantidades mayores de aire y materia fecal.

Wagensteen y sus colegas ${ }^{11}$ refieren que debido a que las tenias rodean la base del apéndice y dan continencia a la unión ceco-apendicular, eventualmente por alteraciones del desarrollo como carencia de una de ellas, no lo hacen dejando la entrada hacia la luz del apéndice cecal, amplia, laxa y abierta para que bolos fecales entren, bloquen la luz y causen la inflamación.

Arlisss y Holgersen ${ }^{8}$ proponen que cuando la perforación se localiza en el base apendicular, se explicaría con base a los principios de la ley de Laplace; encontrando que la presión interna (presión intracolónica) es siempre mayor que en el exterior (espacio pericolónico) y que la diferencia de presiones se incrementa cuando

Cuadro 1. Apendicitis asociada a la enfermedad de Hirschsprung. Revisión de la literatura

\begin{tabular}{|c|c|c|c|c|c|}
\hline Autor & Edad & Sexo & País & Extensión & Desenlace \\
\hline Sahnoun et $\mathrm{al}^{(3)}$ & 3 días & Niña & Túnez & Recto sigmoides & Murió \\
\hline Newman et $\mathrm{al}^{(7)}$ & 4 meses & Varón & EU & Agangliosis coli & Vivió \\
\hline Arliss et $\mathrm{al}^{(8)}$ & 7 días & Varón & EU & Recto sigmoides & Vivió \\
\hline Sarioglu et al ${ }^{(9)}$ & 40 días & Varón & Turquía & Agangliosis coli & Murió \\
\hline Sarioglu et $\mathrm{al}^{(9)}$ & 27 días & Niña & Turquía & Colon descendente & Murió \\
\hline Stiefel et al ${ }^{(10)}$ & 3 semanas & Niña & Suiza & Agangliosis coli & Murió \\
\hline Wyllie ${ }^{(12)}$ & 7 días & $i$ & EU & No registrado & $i$ \\
\hline Martin et $\mathrm{al}^{(13)}$ & 1 día & Varón & EU & Colon transverso & Vivió \\
\hline Martin et $\mathrm{al}^{(13)}$ & 15 días & Varón & EU & Angulo esplénico & Murió \\
\hline Martin et $\mathrm{al}^{(13)}$ & 35 días & Varón & EU & Angulo esplénico & Murió \\
\hline Asch et $\mathrm{al}^{(14)}$ & Desconocida & $i$ & EU & Agangliosis coli & $i$ \\
\hline Asch et $\mathrm{al}^{(14)}$ & Desconocida & $i$ & EU & Agangliosis coli & $i$ \\
\hline Swenson et $\mathrm{al}^{(15)}$ & $i$ & $i$ & EU & Agangliosis coli & $i$ \\
\hline Gastrin et $\mathrm{al}^{(16)}$ & 11días & Varón & EU & mitad distal colon & Vivió \\
\hline Baeza et al & 12 meses & Varón & México & Recto sigmoides & Vivió \\
\hline
\end{tabular}

EU: Estados Unidos. 
disminuye el radio de la superficie (estrechamiento o disminución del radio ocasionada por la disminución del lumen apendicular). La aplicación fisiológica de la ley explicaría que sumando los aumentos crecientes de la presión interna del colon, más el incremento de la misma, una vez que disminuye de calibre el diámetro del intestino grueso, genera en respuesta una mayor presión que al encontrarse con una estructura tubular y sin salida, como es el caso del apéndice cecal, se precipita la perforación. Sin embargo, no existe explicación alguna cuando la perforación aparece en el tercio medio o en el extremo proximal de la estructura. $^{9}$

En la revisión de la literatura sólo existen reportados, hasta el día de hoy, 14 casos de apendicitis con enfermedad de Hirschsprung ${ }^{12-16}$ y la condición predomina en el sexo masculino, es más común la aparición en el periodo neonatal y de afectación mayoritaria de la totalidad del colon, la que se presentó en seis de los 14 casos conocidos. Diez del total fueron publicados en Estados Unidos. De los doce casos en que conocimos el pronóstico, la mitad murió.

\section{REFERENCIAS}

1. Reyes MM. Enfermedad de Hirschsprung: neurocristopatía de la migración y la diferenciación celular. Rev Gastroenterol Mex 1997;62:287-292.

2. Anderson RB, Newgreen DF, Ypung HM. Neural crest and the development of the enteric nervous system. Adv Exp Med Biol. 2006;589:181-196.
3. Oelberg DG, Dominguez R, Hebert AA. Neurocristopathy syndrome: Review of four cases. Pediatr Dermatol. 1990;7:87-92.

4. Sahanoun L, Kitar M, Maazoun K, Ksia A, Chahed J, Mekki $\mathrm{M}, \mathrm{y}$ cols. Hirschsprung's disease presenting as neonatal appendicitis. J Neonatal Surg. 2013;2:25.

5. Sieber WK. Hirschsprung's disease. In: Welch KJ, Randolph JG, Ravitch MM, O'Neill JA, Rowe MI. Pediatric Surgery. Volume II, Year Book Med Pub 4th edition, Chicago 1986; p1000.

6. Baeza, HC, Guido RO, González JAG, Rojas EA. Apendicitis en niños menores de tres años. Rev Gastroenterol Mex. 1994;59:304-307.

7. Newman B, Nussbaum A, Kirkpatrick JA, Colodny A. Appendiceal perforation, pneumoperitoneum, and Hirschsprung's disease. J Pediatr Surg. 1988;23:854-56.

8. Arliss J, Holgersen LO. Neonatal appendiceal perforation and Hirschsprung's disease. J Pediatr Surg. 1990;25:694-5.

9. Sarioglu A, Tanyel FC, Buyukpamukcu N, Hicsonmez A. Appendiceal perforation: A potentially lethal initial mode of presentation of Hirschsprung's disease. J Pediatr Surg. 1997;32:123-124.

10. Stiefel D, Stallmach T, Sacher P. Acute appendicitis in neonates: complication or morbus sui generis. Pediatr Surg Int. 1998;14:122-123.

11. Wangensteen $\mathrm{OH}$, Buirge RE, Dennis C, Wallace RP. Studies in the etiology of acute appendicitis. Ann Surg. 1937;106:910-942 (citado por Sarioglu et al).

12. Willye GG. Course and management of Hirschsprung's disease. Lancet. 1957;2:847-855.

13. Martin LW, Perrin EV. Neonatal perforation of the appendix in association with Hirschsprung's disease. Ann Surg. 1967;166:799-802.

14. Asch MJ, Weitzman JJ, Hays DM. Total colonic agangliosis. Arch Surg 1972;105:74-78.

15. Swenson O, Davidson FZ. Similarities of mechanical intestinal obstruction and aganglionic megacolon in the newborn infant. Review of 64 cases. N Engl J Med. 1960;262:64-67.

16. Gastrin U, Josephson S. Appendiceal perforation and megacolon in the neonatal period. Acta Chir Scand. 1970;136:153-55. 